\title{
Fatal Rupture of Dissecting Anterior Inferior Cerebellar Artery Aneurysm as an Unexpected Complication After Anterior Skull Base Surgery: A Case Report
}

\author{
Eyyub S M AL-BEYATI, Ihsan DOGAN, Agahan UNLU, Melih BOZKURT \\ Ankara University, School of Medicine, Department of Neurosurgery, Ankara, Turkey
}

\section{ABSTRACT}

Aneurysm of the anterior inferior cerebellar artery (AICA) is a very rare entity, and the manifestation and manipulation of such aneurysms remain contentious. In this paper, we report a case where a successful surgery for an olfactory groove meningioma was performed and the patient discharged, but re-admitted to hospital with loss of consciousness and subsequently passed away three hours after re-admission. The patient was diagnosed with subarachnoid hemorrhage. The cause of the fatal subarachnoid hemorrhage was rupture of a new onset AICA dissecting aneurysm which had provided neither clinical nor radiological signs before the operation. We discuss the possible causes of the formation of such aneurysm.

KEYWORDS: Dissecting anterior inferior cerebellar artery aneurysm, latrogenic, Rupture

\section{INTRODUCTION}

A neurysm of the anterior inferior cerebellar artery (AICA) is a very rare entity that makes up less than $1 \%(0.1-$ $0.5 \%)$ of all intracranial aneurysms $(5,7)$. Most are found in the junction between the basilar artery (BA) and AICA. Besides, aneurysms of AICA, even the dissecting ones and notably of the proximal segment are extremely rare (2).

We report a patient who had a total removal of a huge olfactory groove meningioma and was discharged, but readmitted to hospital after a short period of time with altered consciousness that was found to be related to subarachnoid hemorrhage (SAH). The cause of the fatal SAH was rupture of a new onset AICA dissecting aneurysm that provided neither clinical nor radiological signs before the operation.

\section{CASE REPORT}

A 53-year-old female patient was admitted to Ankara University, School of Medicine, Department of Neurosurgery complaining of a headache, loss of vision and personality changes.
Neurological examination revealed visual impairment, and fundoscopic examination revealed papilledema. Magnetic resonance imaging (MRI) revealed an 8-centimeter olfactory groove meningioma (Figure 1A). She had hypertension and diabetes mellitus, with no additional disorders. Four-vessel digital subtraction angiography (DSA) was performed before the operation to determine the possibility of preoperative tumor embolization, and there were no vascular anomalies on the posterior circulation (Figure 1B). Total removal of the tumor was achieved via bifrontal craniotomy (Figure 2). The vision of the patient was improved after the surgery and she was discharged after 10 days.

The patient was re-admitted to the hospital with loss of consciousness one week after the discharge. According to the history obtained from the patient's relatives, she complained of a sudden onset of severe headache, and the loss of consciousness occurred thereafter. The Glasgow Coma Scale score of the patient was 3 at the time of admission. The pupils were dilated and there was no reaction to light. Stiffness of the neck was also observed. Cranial computed tomography 
(CT) revealed a massive SAH and tetra-ventricular hematoma (Figure 3). Also CT-angiography revealed a dissecting aneurysm of left AICA (Figure 4A, B). A ventriculostomy catheter was placed, but the patient passed away three hours after admission.

\section{DISCUSSION}

Dissecting aneurysms of AICA remain obscure regarding the causes, diagnosis and treatments. The case reported here points out the contentious aspects of the reasons of these cases. The most common causes of such a dissection may be discussed in the light of three possibilities: A failure of imaging examinations to demonstrate such an aneurysm during preparation for the operation; spontaneous formation of the aneurysm during follow-up; formation of the aneurysm after an iatrogenic dissection of the artery during angiography.

In this case, we can eliminate the first possibility because the 4 -vessel cerebral DSA was performed just 2 weeks before the operation and revealed no anomalies. The second possibility can be discussed, but the time interval between the event and the first angiography is too short for the formation of such an aneurysm. Thus, the third possibility should be considered as the point of discussion.

Rather than BA alone, most of the reported cases refer to Basilar-Vertebral Artery (VA) dissections. Some hypotheses regarding the formation of such aneurysms have been mentioned in some previous reports, but a definite cause remains obscure (9). Tokimura et al. suggest that anomalies regarding the posterior circulation may contribute to the development of distal AICA aneurysms alongside factors involving the VA (11). They also emphasize the tendency of distal AICA dissecting aneurysms to be located at the anterior pontine segment as a result of stronger hemodynamic stress in the more proximal segments (10). The site of the dissection was another aspect to discuss given that previously reported series demonstrate the dissection site in relatively long vessels, but in our case it was on the AICA. Additionally, the

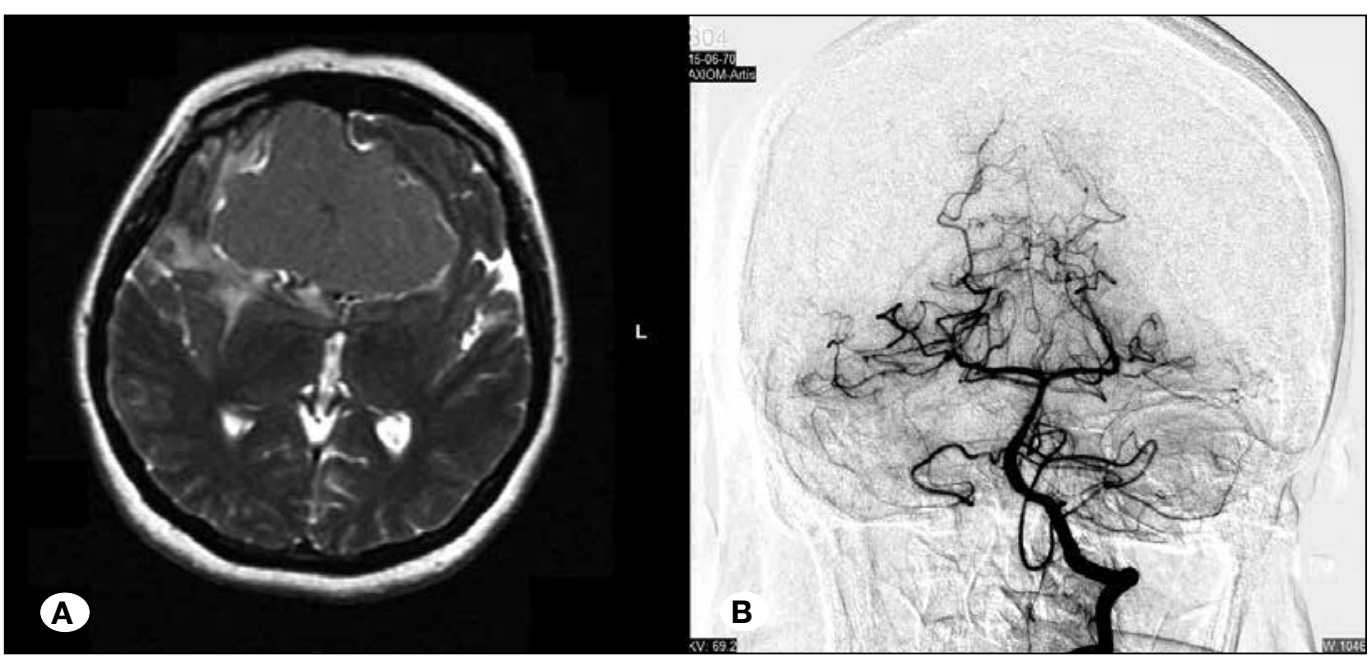

Figure 1: A) Preoperative axial T2W MRI scan showing the case of a huge olfactory groove meningioma.

B) Preoperative four-vessel DSA scan of the patient.

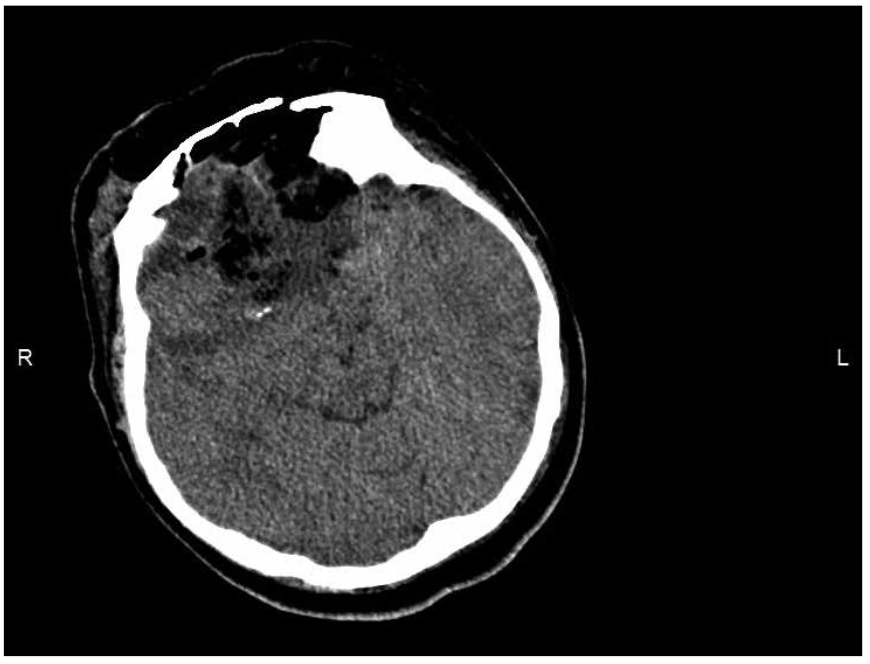

Figure 2: Postoperative CT scan of the patient confirming total removal of the tumor.

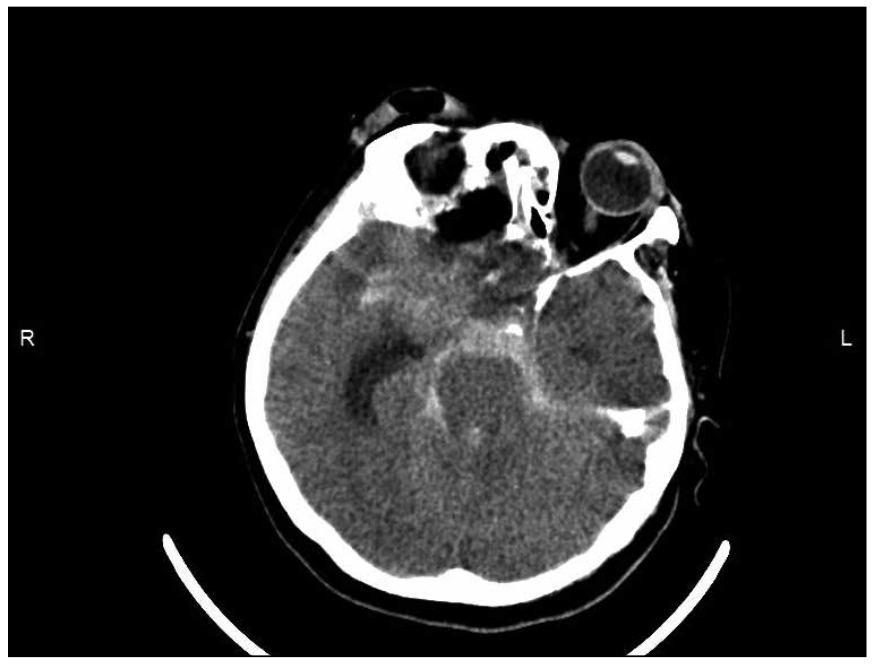

Figure 3: CT scan after re-admission showing massive SAH. 

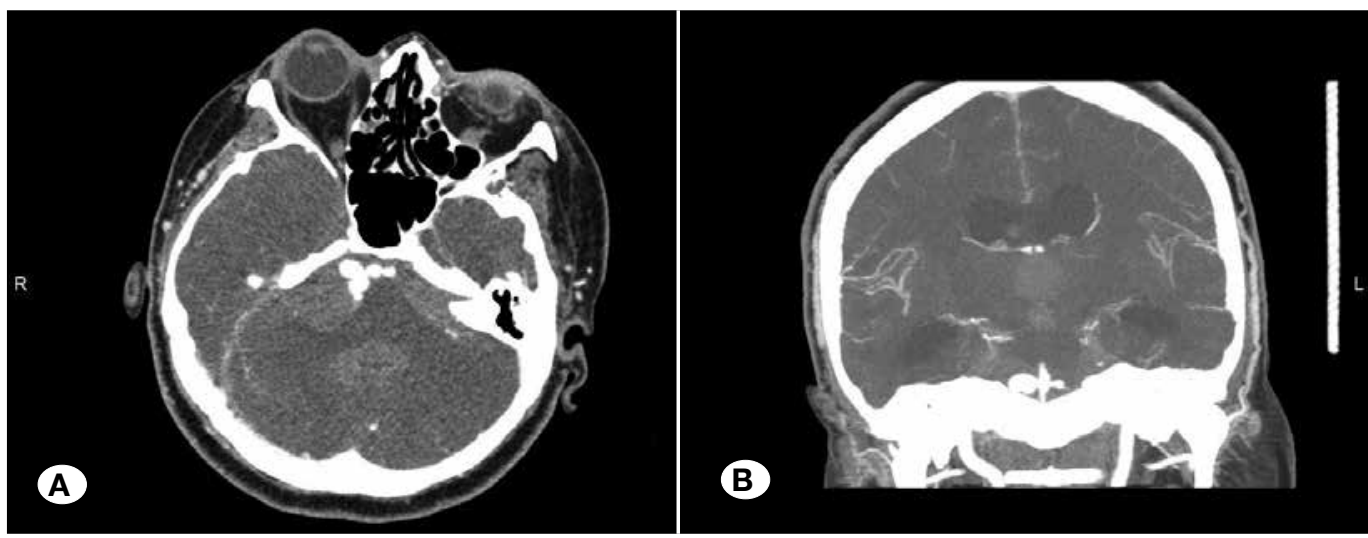

Figure 4: A) Axial and

B) coronal CT-angiography after re-admission showing AICA aneurysm.

dissection was manifested with $\mathrm{SAH}$, not transient ischemic attack or stroke.

Angiography is an invasive procedure that has potential risks, yet it remains the gold standard in the name of vascular examinations. Regarding the iatrogenic dissection, its prevalence was reported to be $0.3 \%$ for diagnostic cerebral angiograms and $0.4 \%$ for all neuroradiological procedures $(1,8)$. The rate of neurological complications related to cerebral angiography was found to vary between $0.9-4$ percent. Also the rates of permanent disability or death were reported to be $0.3-$ $1.3 \%(3,4,6,12)$. There is no statistically significant difference between diagnostic and interventional cerebral angiography, and in our report, the aim of such angiography was only to utilize any possibility of tumor embolization. Interestingly, on the point of clinical indication, Leffers and Wagner reported the highest complication rate after a cerebral angiogram to be found among patients with an indication of a tumor (6). In contrast to the authors' belief that this is a "chance finding", they signify a normal angiogram to be the only factor that significantly increases the risk of neurological complications. This may be valuable enough to underline the role of underestimating such complications in patients with normal angiograms. latrogenic dissections during angiography were reported to be more common in patients with aneurysms.

Cloft et al. reported $11.1 \%$ of dissections to affect the VA (1). They also posited the lack of reported iatrogenic dissections to be related to a tendency to consider such dissections as minor "technical events" rather than complications. Willinsky et al. emphasized the fact that most reports include mainly the complications that occurred within 24 or 24-72 hours of the cerebral angiography (12). In our case, the dissecting aneurysm ruptured approximately 2 weeks after the angiography, and if being an iatrogenic complication is more than a speculation, longer follow-up time is needed and the process should be reviewed for such patients.

\section{CONCLUSION}

We report the first case of a new onset, fatal, possible iatrogenic dissecting aneurysm of the anterior inferior cerebellar artery. The case itself brings all the diagnosis, surgical and postoperative processes into question, and may bring more notions into discussion and guide future research.

\section{- REFERENCES}

1. Cloft HJ, Jensen ME, Kallmes DF, Dion JE: Arterial dissections complicating cerebral angiography and cerebrovascular interventions. AJNR Am J Neuroradiology 21: 541-545, 2000

2. Gonzalez LF, Alexander MJ, McDougall CG, Spetzler RF: Anteroinferior cerebellar artery aneurysms. Surgical approaches and outcomes-a review of 34 cases. Neurosurgery 55: 1025-1033, 2004

3. Heiserman JE, Dean BL, Hodak JA, Flom RA, Bird CR, Drayer BP, Fram EK: Neurologic complications of cerebral angiography. AJNR Am J Neuroradiology 15:1401-1407, 1994

4. Johnston DC, Chapman KM, Goldstein LB: Low rate of complications of cerebral angiography in routine clinical practice. Neurology 57: 2012-2014, 2001

5. Kamano S, Kirino T, Mizuno S: Intrameatal aneurysm. Neurochirurgia (Stuttg) 29: 28-30, 1986

6. Leffers AM, Wagner A: Neurologic complications of cerebral angiography a retrospective study of complication rate and patient risk factors. Acta Radiol 41:204-210, 2000

7. Locksley HB: Natural history of subarachnoid hemorrhage, intracranial aneurysms and arteriovenous malformations. J Neurosurg 25: 321-368, 1966

8. Olivecrona $\mathrm{H}$ : Complications of cerebral angiography. Neuroradiology 1977: 175-181, 1977

9. Schievink WI, Mokri B, Piepgras DG: Angiographic frequency of saccular intracranial aneurysms in patients with spontaneous cervical artery dissection. J Neurosurg 76: 62-66, 1992

10. Tokimura $H$, Ishigami $T$, Yamahata $H$, Yonezawa $H$, Yokoyama S, Haruzono A, Obara S, Nishimuta Y, Nagayama T, Hirahara $\mathrm{K}$, Kamezawa T, Sugata S, Arita K: Clinical presentation and treatment of distal anterior inferior cerebellar artery aneurysms. Neurosurg Rev 35(4): 497-503, 2012

11. Tokimura $H$, Yamahata $H$, Kamezawa T, Tajitsu K, Nagayama T, Sugata S, Takiguchi K, Taniguchi A, Niiro M, Hirahara K, Takasaki K, Oyoshi T, Arita K: Clinical presentation and treatment of distal posterior inferior cerebellar artery aneurysms. Neurosurg Rev 34: 57-67, 2011

12. Willinsky RA, Taylor SM, TerBrugge K, Farb RI, Tomlinson G, Montanera W: Neurologic complications of cerebral angiography; prospective analysis of 2,899 procedures and review of the literature. Radiology 227: 522-528, 2003 